\title{
The solar and interplanetary causes of the recent minimum in geomagnetic activity (MGA23): a combination of midlatitude small coronal holes, low IMF $B_{Z}$ variances, low solar wind speeds and low solar magnetic fields
}

\author{
B. T. Tsurutani ${ }^{1,2}$, E. Echer ${ }^{1}$, and W. D. Gonzalez ${ }^{1}$ \\ ${ }^{1}$ Instituto Nacional de Pesquisas Espaciais, Sao Jose dos Campos, SP, Brazil \\ ${ }^{2}$ Jet Propulsion Laboratory, California Institute of Technology, Pasadena, CA, USA
}

Received: 8 November 2010 - Revised: 31 March 2011 - Accepted: 11 April 2011 - Published: 16 May 2011

\begin{abstract}
Minima in geomagnetic activity (MGA) at Earth at the ends of SC23 and SC22 have been identified. The two MGAs (called MGA23 and MGA22, respectively) were present in 2009 and 1997, delayed from the sunspot number minima in 2008 and 1996 by $\sim 1 / 2-1$ years. Part of the solar and interplanetary causes of the MGAs were exceptionally low solar (and thus low interplanetary) magnetic fields. Another important factor in MGA23 was the disappearance of equatorial and low latitude coronal holes and the appearance of midlatitude coronal holes. The location of the holes relative to the ecliptic plane led to low solar wind speeds and low IMF $\left(B_{\mathrm{Z}}\right)$ variances $\left(\sigma_{B z}^{2}\right)$ and normalized variances $\left(\sigma_{B z}^{2} / B_{0}^{2}\right)$ at Earth, with concomitant reduced solar wind-magnetospheric energy coupling. One result was the lowest ap indices in the history of ap recording. The results presented here are used to comment on the possible solar and interplanetary causes of the low geomagnetic activity that occurred during the Maunder Minimum.
\end{abstract}

Keywords. Magnetospheric physics (Solar wind-magnetosphere interactions)

\section{Introduction}

The recent solar cycle was the longest with the deepest minimum in sunspot number $\left(R_{\mathrm{Z}}\right)$ values in the space exploration era (Hathaway, 2010). The deep minimum is also reflected in several solar irradiance observations, such as total solar irradiance, UV and EUV irradiance, and radio flux at $10.7 \mathrm{~cm}$ wavelengths (Solomon et al., 2010; Hathaway, 2010).

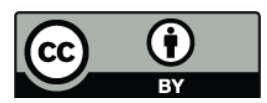

Correspondence to: B. T. Tsurutani

(bruce.tsurutani@jpl.nasa.gov)
It is well known that the transference of solar wind energy to the magnetosphere depends on several interplanetary parameters: the interplanetary magnetic field magnitude $\left(B_{0}\right)$, the southward component of this field $\left(B_{\mathrm{Z}}\right)$ and solar wind speed ( $\left.V_{\text {SW }}\right)$ (Tsurutani and Meng, 1972; Akasofu, 1981; Gonzalez et al., 1994, 1999; Tsurutani et al., 1988; 1995, 2006; Echer et al., 2005, 2008). The purpose of this paper is to identify when the minimum in geomagnetic activity (MGA) occurs relative to the 1996 and 2008 "official" solar (sunspot) minima, and to identify specific solar and interplanetary features that lead to the MGAs. What has not been done before is to examine the causes of variations of magnetic field magnitude, the IMF $B_{\mathrm{Z}}$ variations and solar wind velocity variations separately during and near solar minima. We will show that when one takes this more general approach, the results are quite surprising.

\section{Data analyses}

Solar data such as the sunspot number $\left(R_{\mathrm{Z}}\right)$, mean magnetic field magnitude of the sun $\left(B_{\text {sun }}\right)$ (Scherrer et al., 1977) were obtained from the Sunspot Index Data Center, Brussels and the Stanford Wilcox Solar Observatory websites (http: //wso.stanford.edu/(http://sidc.oma.be/), respectively. The solar wind parameters such as the solar wind velocity $\left(V_{\mathrm{sw}}\right)$, the interplanetary magnetic field (IMF) magnitude $\left(B_{0}\right)$ and the geocentric solar magnetospheric (GSM) $B_{\mathrm{Z}}$ components, as well as the geomagnetic ap indices (e.g., Rostoker, 1972) were obtained from the NASA Goddard OMNI web database (http:/omniweb.gsfc.nasa.gov/). The Oulu, Finland cosmic ray flux was obtained from the Oulu University website (http://cosmicrays.oulu.fi/). Coronal hole maps were obtained from the National Solar Observatory homepage (http: //solis.nso.edu/vsm/). 


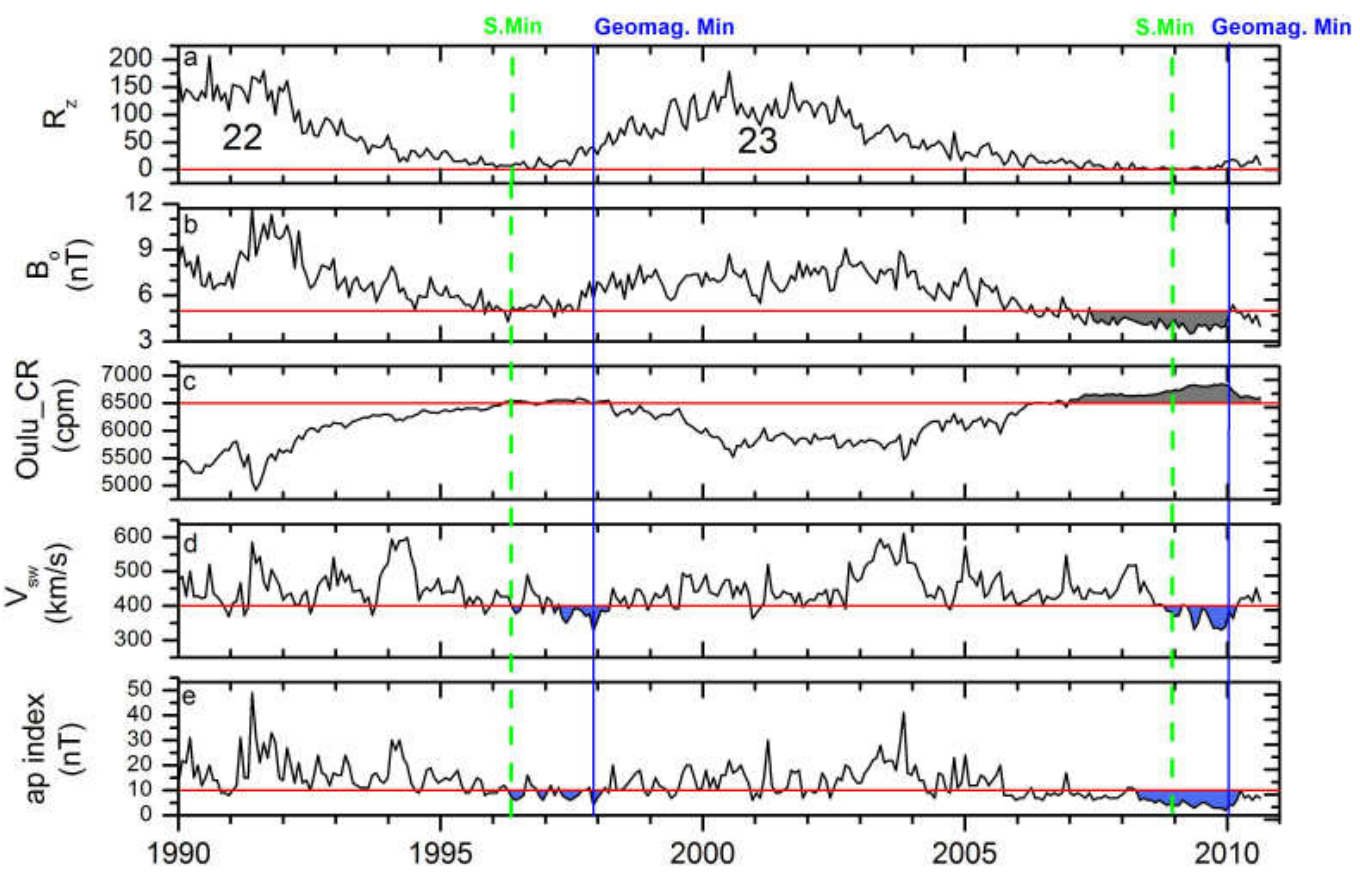

Fig. 1. Solar, interplanetary, cosmic ray and geomagnetic activity data for SC22 and 23. A dashed vertical green line indicates the official sunspot minima. A vertical solid line shows the geomagnetic activity minima on Earth. Horizontal (arbitrary) red lines are shown for $R_{\mathrm{Z}}=0, B_{0}=5 \mathrm{nT}, \mathrm{CR}=6500 \mathrm{cpm}, V_{\mathrm{SW}}=400 \mathrm{~km} \mathrm{~s}^{-1}$ and ap $=10 \mathrm{nT}$. The shaded regions in the ap panel are used to define the minimum geomagnetic activity (MGA) intervals, MGA22 and MGA23.

Both the measured IMF $B_{\mathrm{Z}}$ and the calculated epsilon parameters (Perrault and Akasofu, 1978) are expressed using geocentric solar magnetosphere (GSM) coordinates. In GSM coordinates, the $\mathrm{x}$-axis points from the Earth towards the sun, the $y$-axis is defined by $\boldsymbol{y}=(\boldsymbol{M} \times \boldsymbol{x}) /|\boldsymbol{M} \times \boldsymbol{x}|$ where $\boldsymbol{M}$ is the magnetic south polar vector. The z-axis completes the righthand system $(\boldsymbol{x} \times \boldsymbol{y}=\boldsymbol{z})$.

\section{Nested variances and normalized variances}

A quantitative measure of the interplanetary Alfvén wave (Belcher and Davis Jr., 1971) intensities is provided by calculating the "nested variances" of the magnetic field $B_{\mathrm{Z}}$ component (Tsurutani et al., 1982, 2011; Echer et al., 2011). Only the $B_{\mathrm{Z}}$ component and variances are shown because this is the most important component leading to geomagnetic activity at Earth. 1-min average magnetic field data were used to calculate the variances. $30-\mathrm{min}, 1-\mathrm{h}$ and 3 -h variances were calculated and then were used to make 3-h averages of the quantities. We show only the 3 -h average values in this paper. These variances are called "nested" because the 3 -h averages of 30-min variance have values less than the 3-h averages of 1-h variance (for the same time interval), and so on. The lowest time scale variance is "nested" inside the value of the next higher time scale variance, etc.

What is useful about this method of data display? The variances are easy to calculate and display. Furthermore, the variances give the amount of wave power for frequencies up to the time value. For example, the 3 -h average 30 -min variance values give the average wave power occurring in the 1-min (the minimum time scale used in the construction of the variances) to 30 -min wave period range. The 1-h variance values give the wave power occurring between 1-min to 1 -h wave period range. If one subtracts the 30 -min variance value from the 1-h variance value, the resultant value is the amount of wave power which was present for wave periods between 30-min and 1-h. The variances can thus be used to determine an average wave power and a low-resolution power spectrum.

Because the ratio of the Alfvénic fluctuation amplitude to the ambient magnetic field strength $B_{0}$ (quantity squared) is an important quantity for particle scattering, the quantity $\sigma_{\mathrm{z}}^{2} / B_{0}^{2}$, the "normalized" variances, have also been calculated. The normalized $B_{\mathrm{Z}}$ variances and $B_{\mathrm{Z}}$ variances will both be shown during a variety of solar wind conditions.

\section{Results}

Figure 1 shows, from top to bottom, the sunspot number $\left(R_{\mathrm{Z}}\right)$, the $1 \mathrm{AU}$ interplanetary magnetic field (IMF) magnitude, $B_{0}$, the Oulu, Finland cosmic ray $(\mathrm{CR})$ count rate (the local vertical geomagnetic cutoff rigidity is $\sim 0.8 \mathrm{GeV}$ ), the solar wind speed $V_{\mathrm{sw}}$, and the ap geomagnetic index. All values displayed are 27-day averages. The data extend from 1 January 1990 to 31 August 2010, covering the last $\sim 1 \frac{1}{2}$ 
solar cycles. The focus of our discussion will be the time intervals near the last two solar minima. Vertical dashed green lines give the official dates of the solar minima between cycle 22 and 23 (hereafter called the SC22 minimum) and cycle 23 and 24 (the SC23 minimum). The sunspot minimum dates were obtained from Hathaway (2010). Vertical blue lines give the geomagnetic ap index minima. We call attention to the long delay of the geomagnetic activity minima from the sunspot minima.

The horizontal red lines have been added to the figure to guide the reader. From top to bottom the lines are the zero value for $R_{\mathrm{z}}, 5 \mathrm{nT}$ for $B_{0}, 6500$ counts/min for the Oulu cosmic ray flux, $400 \mathrm{~km} \mathrm{~s}^{-1}$ for $V_{\mathrm{SW}}$ and $10 \mathrm{nT}$ for ap.

Figure 1 shows the solar cycle that has just ended (SC23) extended from 1996 to 2008 and is therefore the longest in the space era (12.6 years). The length of solar cycles 20 through 22 were $11.7,10.3$ and 9.7 years, respectively. The solar minimum sunspot number average for the last cycle is 1.7, which is also considerably lower than the other minima: 12.2, 12.3 and 8.0, respectively (not shown).

Using the horizontal red lines, the values below $\left(B_{0}, V_{\mathrm{sw}}\right.$ and ap) and above (CR flux) the lines near the SC23 minimum have been shaded for emphases. The same shading has been done for the interval around the SC22 minimum. It can be noted that $B_{0}, V_{\text {sw }}$ and the ap index values for the SC23 minimum are considerably lower than the SC22 minimum values.

Although the IMF $B_{0}$ and Oulu CR flux shaded regions are symmetric in time about the official solar minimum (in late 2008), Fig. 1 shows that the $B_{0}$ minimum is weighted more towards 2009 and the CR flux peak is not reached until the beginning of 2010. What is particularly interesting in the figure is that the $V_{\mathrm{SW}}$ and ap 27-day averages are even more asymmetric about the solar minimum time. Both quantities have minima near the end of 2009/beginning of 2010.

For the SC22 minimum, it is noted that both $V_{\mathrm{SW}}$ and the ap indices shaded minima are similarly delayed in time from the official sunspot minimum, just as in the case of the SC23 minimum. The $B_{0}$ values are symmetric about the minimum while the CR flux is again delayed.

The focus of this paper is the minima in geomagnetic activity (MGA), so we will use the ap shaded regions as foci for further discussion. We select the MGA23 interval from the ap shading as being from day 97, 2008 to day 95, 2010 and the MGA22 interval from day 106, 1996 to day 23, 1998. The onset and end times are somewhat arbitrary, depending on what are chosen for the horizontal cutoff values. However the main point is that the MGAs are delayed from the SC minima.

Table 1 gives the ap index, $V_{\mathrm{sw}}, B_{0}$ average and CR flux values for the selected MGA23 interval and the selected MGA22 interval. For the MGA23 interval, the ap, $V_{\mathrm{sw}}$ and $B_{0}$ averages were $5.0 \mathrm{nT}, 390 \mathrm{~km} \mathrm{~s}^{-1}$, and $4.2 \mathrm{nT}$, in comparison to $8.7 \mathrm{nT}, 395 \mathrm{~km} \mathrm{~s}^{-1}$, and $5.5 \mathrm{nT}$ for the MGA22 inter-
Table 1. Average values for MGA23 and MGA22.

\begin{tabular}{cll}
\hline & MGA23 & MGA22 \\
\hline Time MGA & $097 / 2008-095 / 2010$ & $106 / 1996-023 / 1998$ \\
ap & $5.0 \pm 1.9 \mathrm{nT}$ & $8.7 \pm 2.6 \mathrm{nT}$ \\
$V_{\text {sw }}$ & $390.2 \pm 43.6 \mathrm{~km} \mathrm{~s}^{-1}$ & $395.0 \pm 34.8 \mathrm{~km} \mathrm{~s}^{-1}$ \\
$B_{0}$ & $4.2 \pm 0.4 \mathrm{nT}$ & $5.5 \pm 0.7 \mathrm{nT}$ \\
CR & $6739.4 \pm 78.4 \mathrm{cpm}$ & $6531.3 \pm 28.8 \mathrm{cpm}$ \\
\hline
\end{tabular}

val. For MGA23, the Oulu CR flux values were $6739 \mathrm{cpm}$ compared to $6532 \mathrm{cpm}$ for MGA22.

During MGA23, the $B_{0}$, Oulu CR flux, $V_{\mathrm{SW}}$ and ap averages are extreme values for the space era (not shown to conserve space). The values of $B_{0}, V_{\mathrm{SW}}$ and ap are extreme minima while that of the Oulu CR fluxes is a maximum. The ap index is a record low value for the history of the index monitoring.

Figure 2 shows the solar wind velocity $V_{\mathrm{SW}}$, the interplanetary magnetic field $B_{0}$, the solar wind epsilon parameter and geomagnetic activity ap index for the MGA23 interval. The data are shown as daily averages. The epsilon parameter (Perrault and Akasofu, 1978) is given by $\varepsilon=V_{\mathrm{SW}} B_{0}^{2} \sin ^{4}(\theta / 2)$, where $\theta$ is the clock angle of the interplanetary magnetic field. Linear fits were made to these solar wind and geomagnetic activity parameters. These are shown by black lines. The previous horizontal red lines from Fig. 1 are indicated again.

The ap peak values are asymmetric around SC23 minimum (the green vertical line). There are many large peak ap values $>10 \mathrm{nT}$ prior to the SC23 minimum and very few large peaks after the SC23 minimum. The largest peak ap values (days $\sim 115, \sim 255$ and $\sim 285$ ) occur during high speed stream intervals. The average ap is $\sim 5 \mathrm{nT}$. There is a small negative trend of $-0.005 \mathrm{nT} \mathrm{day}^{-1}$ with an $R$ of -0.25 .

There is a decrease in the peak velocities in the high speed streams with increasing time. The velocities are greater than $600 \mathrm{~km} \mathrm{~s}^{-1}$ on days $\sim 99,101,126,167,170,179,195,197$, 205, 223-4, 276-8, and 303-5, 2008, prior to the SC23 minimum. After the SC23 minimum until the end of the interval there are no peak speeds higher than $600 \mathrm{~km} \mathrm{~s}^{-1}$. The linear fit has an $R$ value of -0.4 with a slope of $-0.15 \mathrm{~km} \mathrm{~s}^{-1}$-day.

The interplanetary $B_{0}$ average is essentially constant with an average value of $4.2 \mathrm{nT}$ over the $\sim 2$ years. There is a very small (positive) slope of $5 \times 10^{-4} \mathrm{nT} \mathrm{day}^{-1}$ with an $R$ of $\sim 0.1$. The same is true for the epsilon value. The average value is $5 \times 10^{10} \mathrm{~W}$, with a very small slope of $2 \times 10^{7} \mathrm{~W} \mathrm{day}^{-1}$ and an $R$ of 0.05 . 


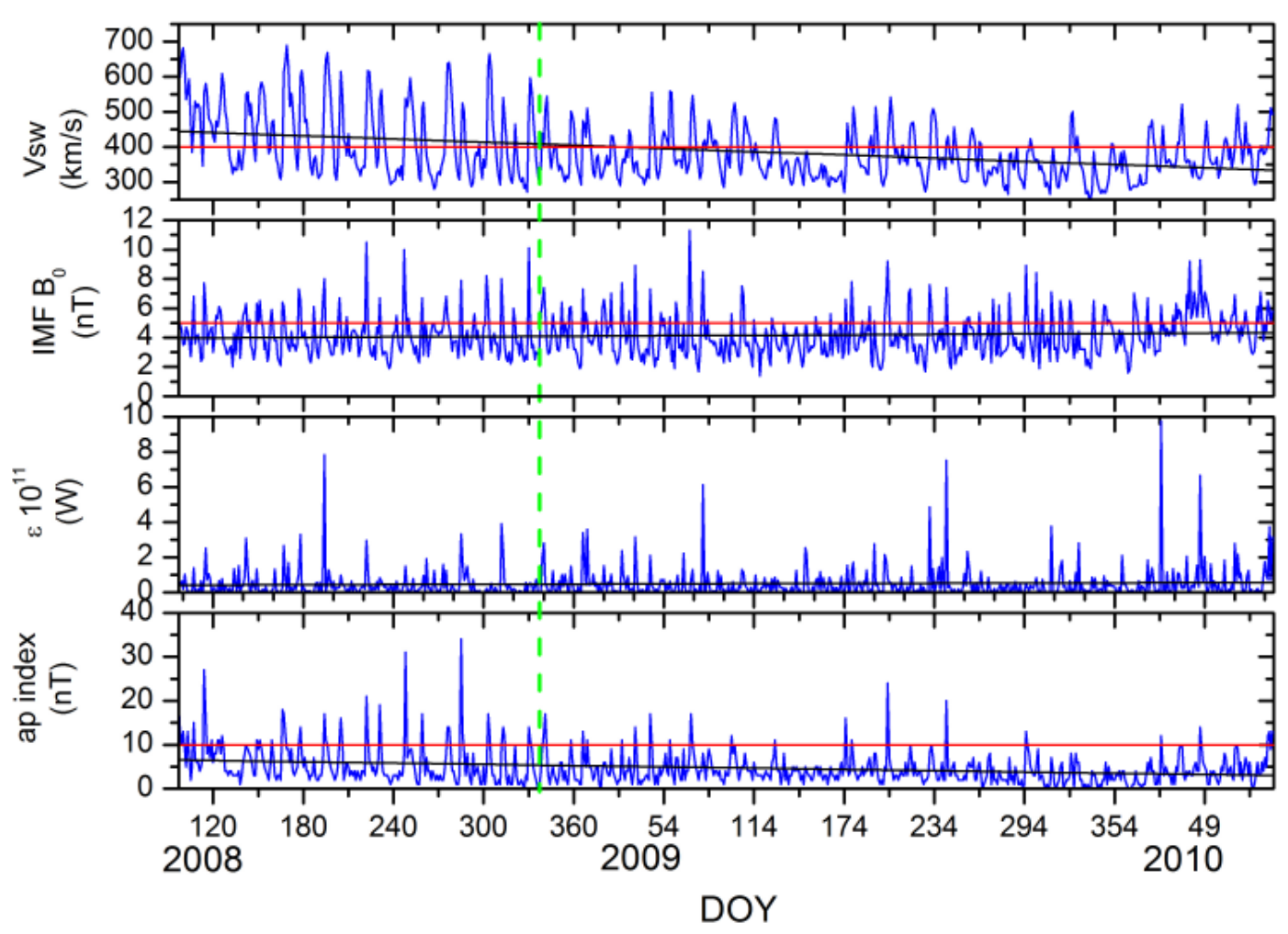

Fig. 2. An expanded view of MGA23. The same horizontal red lines shown in Fig. 1 are duplicated here for reference. The black lines give linear regression fits to the parameters.

\subsection{Low interplanetary magnetic fields}

Why are the interplanetary magnetic fields so low during the MGA23 interval? This is examined in Fig. 3. From the top, the panels are: the sunspot number $R_{\mathrm{z}}$, the Stanford solar mean field $B_{\text {sun }}$ and the IMF $B_{0}$. The sunspot minimum is again given by a green dashed line and the MGA interval is indicated by a bracket at the top. The very low interplanetary $B_{0}$ during the MGA is well correlated with the low Stanford solar magnetic fields $B_{\text {sun }}$.

\subsection{Low magnetic variances and normalized variances at Earth}

Figure 4 shows from top to bottom: $V_{\mathrm{SW}}$, GSM IMF $B_{\mathrm{Z}}$, $B_{\mathrm{Z}}$ nested variances $\left(\sigma_{\mathrm{Z}}^{2}\right), B_{\mathrm{Z}}$ normalized nested variances $\left(\sigma_{\mathrm{Z}}^{2} / B_{0}^{2}\right)$ and ap for the MGA23 interval. $V_{\mathrm{SW}}$ and ap have been repeated here for reference. The time averaging intervals have been decreased to improve the viewing of the details of the parameters. $V_{\mathrm{SW}}$ and $B_{\mathrm{Z}}$ are given in 1-min averages, $\sigma_{\mathrm{z}}^{2}$ and $\sigma_{\mathrm{z}}^{2} / B_{0}^{2}$ are still 3-h averages and ap is given in one-day averages.

It can be noted that there are general trends in the $\sigma_{\mathrm{z}}^{2}$ and $\sigma_{\mathrm{z}}^{2} / B_{0}^{2}$ values which generally follow the solar wind speeds. $\sigma_{\mathrm{z}}^{2}$ and $\sigma_{\mathrm{z}}^{2} / B_{0}^{2}$ are highest at the beginning of the interval, decrease with decreasing solar wind peak speeds and then increase near the end of the interval. To illustrate this quantitatively, we have calculated the average values of $V_{\mathrm{SW}}, B_{\mathrm{Z}}$, $\sigma_{\mathrm{z}}^{2}, \sigma_{\mathrm{z}}^{2} / B_{0}^{2}$ and ap for two separate intervals of Fig. 4, from day 97 to day 365, 2008 and for the entire year of 2009 where there is a lack of high speed streams with peak speeds $>600 \mathrm{~km} \mathrm{~s}^{-1}$ (there is now 1 event on day 58, 2009 which was not present in Fig. 1 due to the time averaging used). For illustrative purposes, we quote only the averages of the 3-h variances. The value for $\sigma_{\mathrm{z}}^{2}$ for the 2008 interval is $2.27 \mathrm{nT}^{2}$. The value for $\sigma z^{2}$ for the 2009 interval is $1.79 \mathrm{nT}^{2}$. It can be noted that the IMF $B_{\mathrm{Z}}$ average values are the same in the two intervals, so there is no obvious large scale trend in that parameter. However for all of the other parameters, the 2008 average values are larger than that in 2009.

\subsection{Solar cycle declining phase IMF $B_{\mathrm{z}}$ variances}

The relationship between solar wind high speed streams and magnetic field $B_{\mathrm{Z}}$ variances, normalized $B_{\mathrm{Z}}$ variances and geomagnetic activity at Earth during the declining phase of the solar cycle have been reported previously (Tsurutani et al., 2011). However it would be useful to repeat part of the results here for two purposes: (1) to illustrate the detailed relationship between the streams, field $B_{\mathrm{Z}}$ variances and geomagnetic activity, and (2) to make an intercomparison between a more geomagnetic active epoch and the MGA23 interval. 


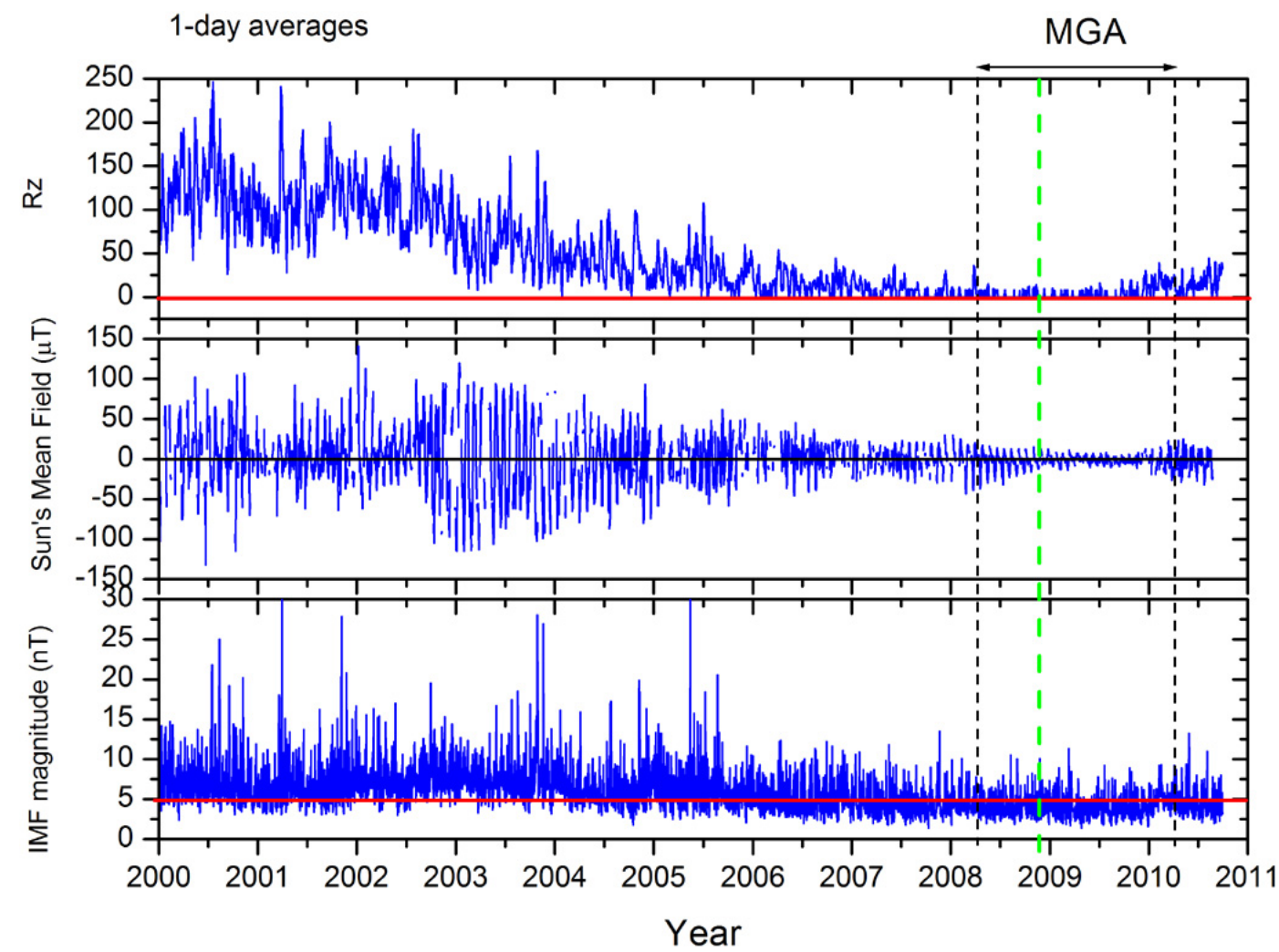

Fig. 3. The top panel is the sunspot number, $R_{\mathrm{Z}}$, the middle panel the solar magnetic field magnitude and the bottom panel the interplanetary magnetic field at $1 \mathrm{AU}$. The sunspot minimum is indicated by the vertical green line. The MGA23 interval is indicated above the graph and by the vertical dashed lines.

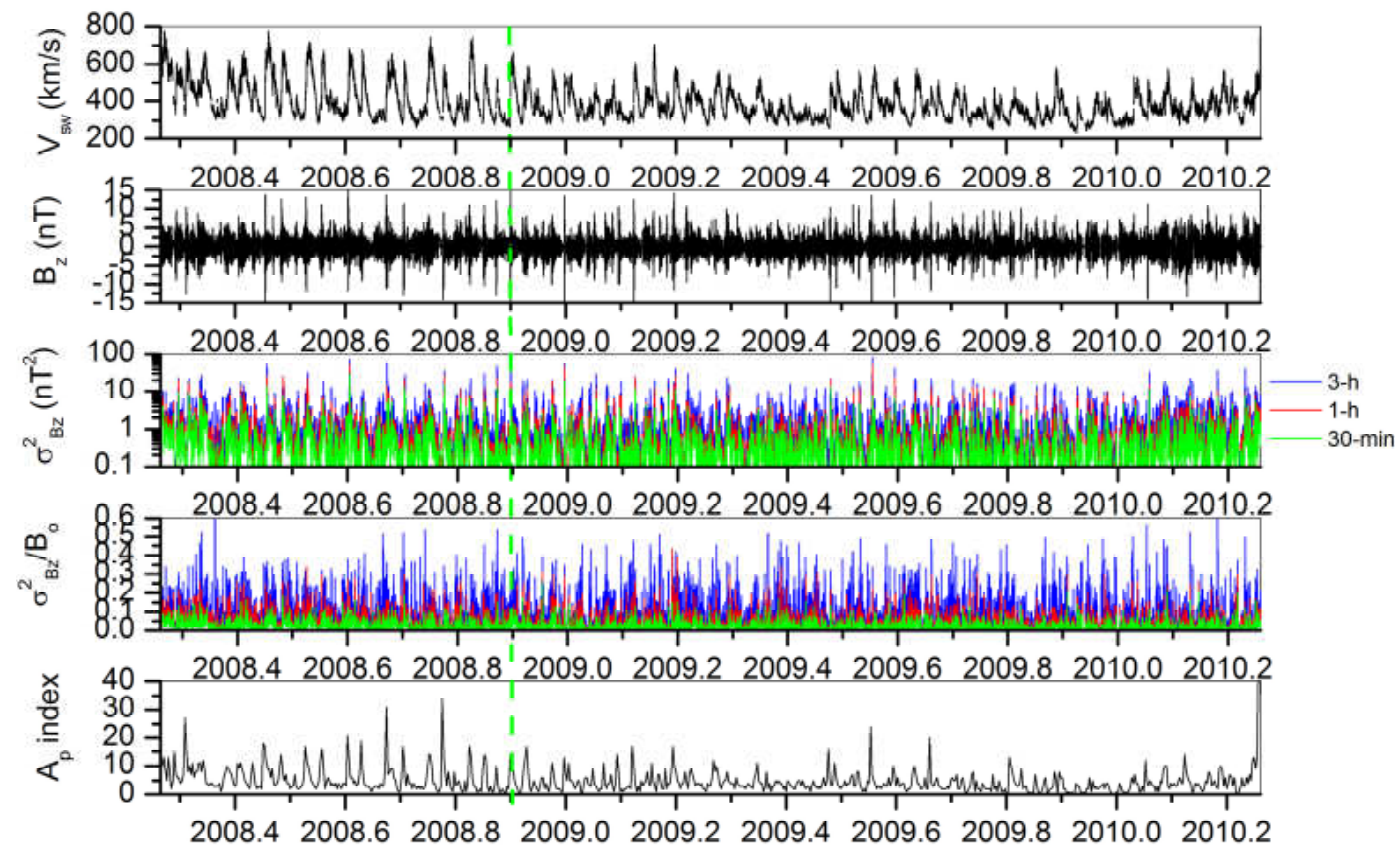

Fig. 4. From top to bottom are: $V_{\mathrm{SW}}$, IMF $B_{\mathrm{Z}}$, the IMF $B_{\mathrm{Z}}$ variances, the IMF normalized $B_{\mathrm{Z}}$ variances and the geomagnetic ap indices. The SC23 minimum is indicated by a vertical dashed green line. 


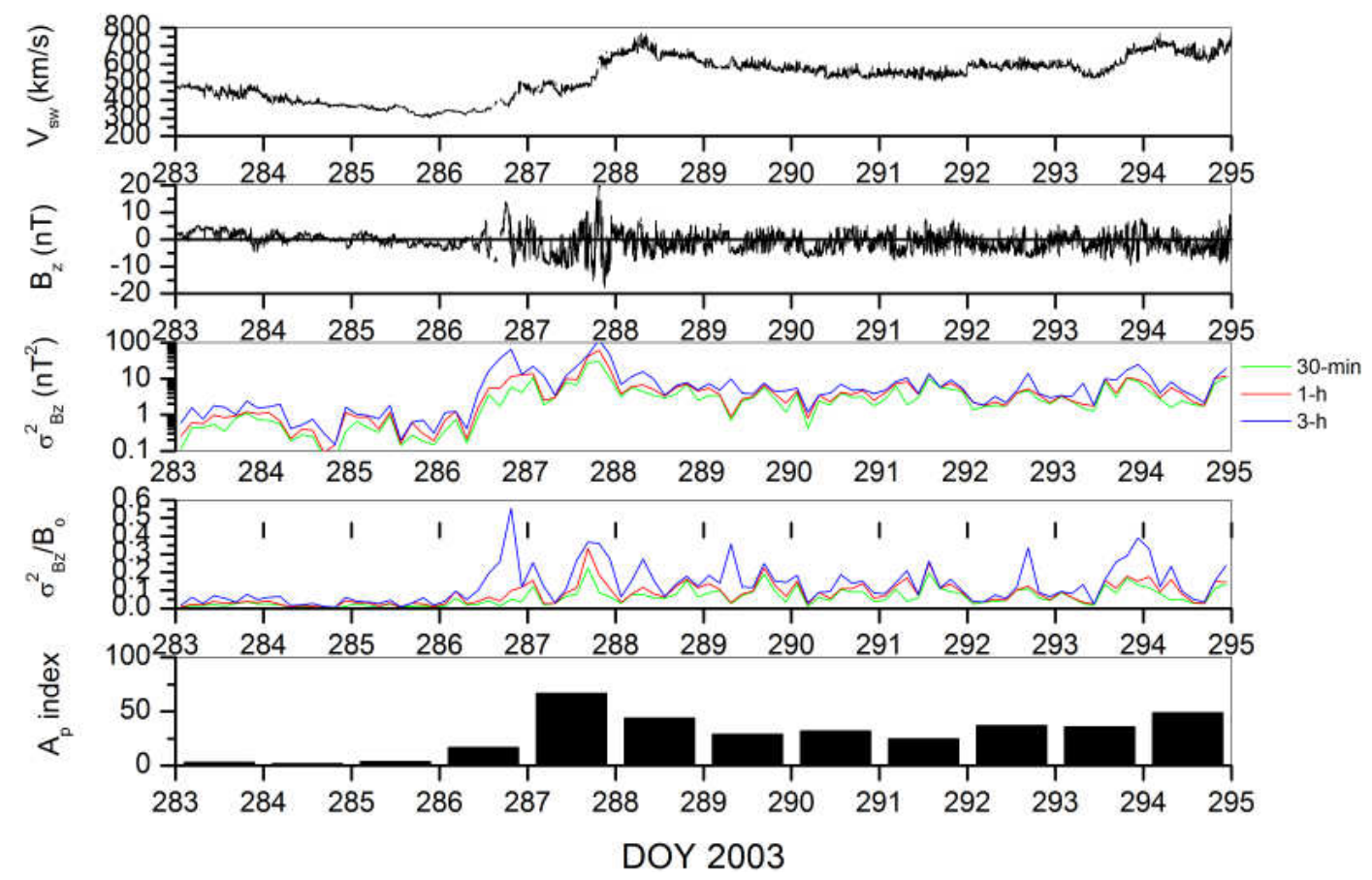

Fig. 5. The same format as in Fig. 4, but for a high speed stream interval during the declining phase of the solar cycle, day $283-295,2003$.

Figure 5 shows data from one of the two Ambleside intervals (high speed stream 2, HSS1) that occurred in the SC23 declining phase interval: days 283-295, 2003. For comparative purposes, the Tsurutani et al. (2011) Fig. 3 has been revised to show the same solar wind, nested variances and geomagnetic activity parameters as was shown in Fig. 4 of this paper. The top panel shows that the high speed stream started at the end of day 286 and reached a peak speed of $\sim 770 \mathrm{~km} \mathrm{~s}^{-1}$ by 07:00 UT on day 288. The speed remained high until the end of day 294 and beyond. The $\sigma_{\mathrm{z}}^{2}$ and $\sigma_{\mathrm{z}}^{2} / B_{0}^{2}$ values are highest at the initial part of the high speed stream (days 286-287). This leading edge of the high speed stream corresponds to the corotating interaction region (CIR, Balogh et al., 1999) where the high speed stream interacted with the upstream slow speed stream. The magnetic fields are compressed by this interaction (not shown to conserve space), giving higher IMF $B_{\mathrm{Z}}$ values (second panel from the top). The variance values are an order of magnitude higher than the corresponding values for days 283-285, the interval prior to the high speed stream. $\sigma_{\mathrm{z}}^{2}$ and $\sigma_{\mathrm{z}}^{2} / B_{0}^{2}$ remain high from days 285 to 295 . The variances and normalized variances are considerably higher than the quiet days prior to the high speed stream.

The bottom panel shows the ap indices. The ap index is the highest on day 287 and the indices remain high throughout the high speed stream. The values are considerably higher from day 287 through 294 than from days 283-285. The average values are $40 \mathrm{nT}$ and $6.5 \mathrm{nT}$, respectively. Why is there a general correlation between the values of $\sigma_{\mathrm{z}}^{2}$ and ap? It has been shown that the southward component of the IMF $B_{\mathrm{Z}}$ leads to magnetic reconnection between interplanetary magnetic field and the magnetopause magnetic field, leading to injection of solar wind energy into the magnetosphere. More details about magnetic reconnection can be found in Dungey (1961), Gonzalez and Mozer (1974), and Gonzalez et al. (1994) and Alfvén waves causing geomagnetic activity in Tsurutani and Gonzalez $(1987,1997)$, and Tsurutani et al. (1995, 2006).

Table 2 gives the $\sigma_{\mathrm{z}}^{2}$ and $\sigma_{\mathrm{z}}^{2} / B_{0}^{2}$ values for the Ambleside (HSS1) interval and that for the MGA23 interval. These are given in column 4 and column 3 , respectively. Note that the variances for HSS1 are 4-6 times higher than the corresponding MGA23 values. The normalized variances are 1.3-2.3 times higher for HSS1 than for MGA23. The corresponding ap indices are given in the bottom row of each of the two sets of values. The ratio of the ap values for HSS 1 to that of MGA23 is $\sim 5.7$, close to the IMF $B_{\mathrm{Z}}$ variance ratio.

Table 2 also gives the $\sigma_{\mathrm{z}}^{2}, \sigma_{\mathrm{z}}^{2} / B_{0}^{2}$ and ap averages for the other Ambleside high speed stream (HSS2= day 314-318, 2003) and MGA22 intervals. These will be discussed later.

\subsection{MGA22}

Figure 6 shows $V_{\mathrm{SW}}$, IMF $B_{\mathrm{z}}, \sigma_{\mathrm{z}}^{2}, \sigma_{\mathrm{z}}^{2} / B_{0}^{2}$ and ap averages for the MGA22 interval. The format is the same as that used in Fig. 4 for MGA23. The solar wind speed is in general high at the beginning of the MGA22 interval from $\sim 1996.6$ to $\sim 1997.2$ and then more-or-less decreases thereafter. There are many streams with peak speeds $>600 \mathrm{~km} \mathrm{~s}^{-1}$ 


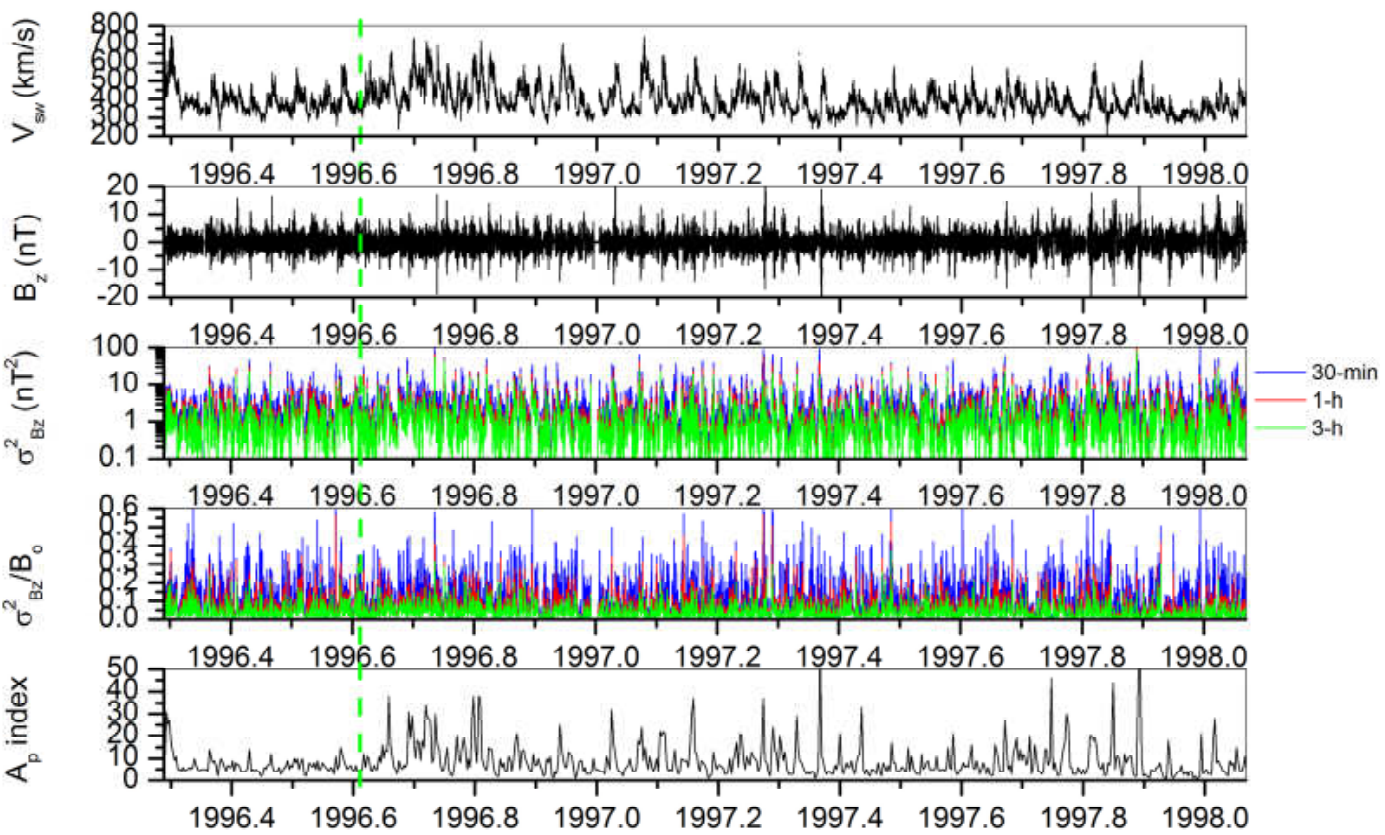

Fig. 6. The same format as in Fig. 4, but for MGA22. The SC22 minimum is indicated by a vertical dashed green line.

Table 2. The $B_{\mathrm{Z}}$ variance and normalized $B_{\mathrm{Z}}$ variances for MGA22, MGA23, Ambleside HSS1 and HSS2 events.

\begin{tabular}{|c|c|c|c|c|}
\hline \multicolumn{5}{|c|}{ Nested variances $\left(\mathrm{nT}^{2}\right)$} \\
\hline & MGA22 & MGA23 & Ambleside HSS 1 & Ambleside HSS2 \\
\hline$\sigma_{30 \min }^{2}$ & $1.37 \pm 2.67$ & $0.055 \pm 1.12$ & $3.47 \pm 4.60$ & $6.23 \pm 3.85$ \\
\hline$\sigma_{60 \min }^{2}$ & $2.15 \pm 4.24$ & $1.04 \pm 2.20$ & $5.00 \pm 7.97$ & $8.30 \pm 5.06$ \\
\hline $\begin{array}{c}\sigma_{180 \min }^{2} \\
\text { ap }\end{array}$ & $\begin{array}{c}3.77 \pm 7.04 \\
9.1\end{array}$ & $\begin{array}{c}2.05 \pm 4.24 \\
5.0\end{array}$ & $\begin{array}{c}8.70 \pm 15.43 \\
28.7\end{array}$ & $\begin{array}{c}12.24 \pm 7.11 \\
41.5\end{array}$ \\
\hline \multicolumn{5}{|c|}{ Normalized nested variances } \\
\hline & MGA22 & MGA23 & Ambleside HSS1 & Ambleside HSS2 \\
\hline$\sigma_{30 \min / B 0^{2}}^{2}$ & $0.04 \pm 0.04$ & $0.03 \pm 0.03$ & $0.06 \pm 0.05$ & $0.09 \pm 0.04$ \\
\hline$\sigma_{60 \min / B 0^{2}}^{2}$ & $0.06 \pm 0.06$ & $0.05 \pm 0.05$ & $0.08 \pm 0.06$ & $0.12 \pm 0.05$ \\
\hline$\sigma_{180 \min / B 0^{2}}^{2}$ & $0.11 \pm 0.10$ & $0.09 \pm 0.09$ & $0.13 \pm 0.11$ & $0.18 \pm 0.09$ \\
\hline
\end{tabular}

(a cluster from day 256 to 265, 293, 297 and 346, 1996 and 28,1997 ) and even $>700 \mathrm{~km} \mathrm{~s}^{-1}$ (day 268, 1998) in the former interval. There are only few high speed streams with $V_{\mathrm{SW}}>600 \mathrm{~km} \mathrm{~s}^{-1}$ beyond 1997.2 (122, 124, 204-5, 2389, 268, 275 and 295, 1996). The averages for the 5 parameters for the first interval are $V_{\mathrm{SW}}=425 \mathrm{~km} \mathrm{~s}^{-1}$, IMF $B_{\mathrm{z}}=-0.17 \mathrm{nT}, \sigma_{\mathrm{z}}^{2}=3.9 \mathrm{nT}^{2}, \sigma_{\mathrm{z}}^{2} / B_{0}^{2}=0.11$ and ap $=9.9 \mathrm{nT}$ and $V_{\mathrm{SW}}=371 \mathrm{~km} \mathrm{~s}^{-1}$, IMF $B_{\mathrm{z}}=-0.21 \mathrm{nT}, \sigma_{\mathrm{z}}^{2}=3.8 \mathrm{nT}^{2}$, $\sigma_{\mathrm{z}}^{2} / B_{0}^{2}=0.10$ and $\mathrm{ap}=8.3 \mathrm{nT}$ for the second interval. The IMF $B_{\mathrm{z}}$ average is slightly more negative during the 1997.3 to 1998.1 interval. The $B_{\mathrm{Z}}$ variances and normalized $B_{\mathrm{Z}}$ variances were about the same in the two intervals. Thus it is most probably the higher solar wind speeds during the 1996.6 to 1997.2 interval that lead to the higher ap indices during that epoch.

Table 2 shows the values of the variances, normalized variances and the ap indices for the full MGA22 interval (column 2). The variances are about double the values of those in the MGA23 interval and about $\sim 2.5$ times smaller than those of the Ambleside HSS1. The normalized variances of MGA22 are greater than those of MGA23. Although the values for the 3-h variances are within $10 \%$ of each other, the 30-min variances of MGA22 are $~ 50 \%$ higher than those of MGA23. The MGA22 normalized variances are $\sim 1.2$ times 


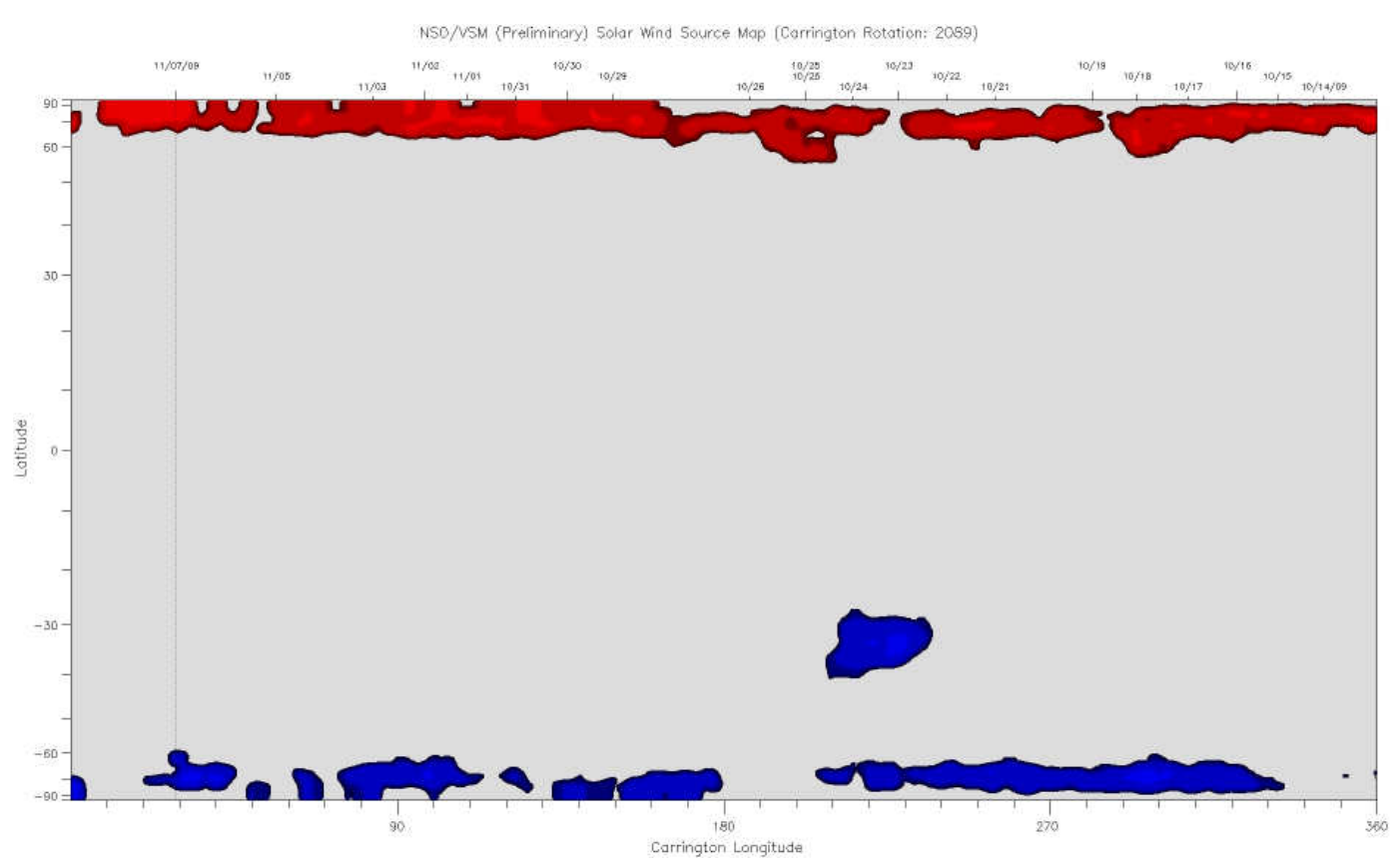

Fig. 7. A midlatitude coronal hole during 2009. Also present are polar coronal holes at both polar regions.

lower than those of HSS1. The Ambleside HSS2 interval had the highest variances of any of the four intervals. The $B_{\mathrm{Z}}$ variances for HSS2 was $\sim 1.4-1.9$ times that of HSS1 while the ap ratio was 1.5 , about the same value.

\subsection{Anomalously low $V_{\mathrm{SW}}$ and changing solar conditions}

Why is the solar wind speed so low during the MGA23 interval? Did anything change on the sun? The nature of solar coronal holes changed as the sunspot minimum was approached and was passed. De Toma (2011) has noted that "by the end of 2008, these low-latitude coronal holes started to close down, and finally disappeared in 2009, while smaller, mid-latitude coronal holes formed in the remnants of cycle 24 active regions, shifting the sources of the solar wind at Earth to higher latitudes". An example of a coronal hole during this minimum geomagnetic activity interval is shown in Fig. 7, taken from the National Solar Observatory for CR2089. The coronal hole is detected from $\sim-30^{\circ}$ to $-35^{\circ}$.

Figure 8 shows a small high speed solar wind stream detected in 1998 during the rising portion of SC23. The format is the same as in Figs. 4 and 6 , but the IMF $B_{0}$ has been added to show the magnetic field compressional effect (CIR) at the front of the high speed stream interval. The CIR is found on days 203-204, in the rising part of the stream speed. The peak variances are found on day 204 more or less timecoincident with the corresponding ap peak value. The higher CIR magnetic fields (and the presence of Alfvénic fluctuations) are the cause of the higher $\sigma_{\mathrm{z}}^{2}$ values. The $\sigma_{\mathrm{z}}^{2} / B_{0}^{2}$ peak values are delayed slightly from the peak $\sigma_{\mathrm{z}}^{2}$ value and occur later on day 204, closer to the peak solar wind speeds. The peak solar wind speed of $\sim 770 \mathrm{~km} \mathrm{~s}^{-1}$ occurs at $\sim 02: 30 \mathrm{UT}$ day 205. This figure is a modification of Fig. 20 in Tsurutani et al. (2006).

The important feature for the reader to recognize here is that $V_{\mathrm{SW}}, \sigma_{\mathrm{z}}^{2}$ and $\sigma_{\mathrm{z}}^{2} / B_{0}^{2}$ values decrease gradually as one goes away from the center (peak speed) of the high speed stream. The average $V_{\text {SW }}$ for days 205 through 208 are 661, 562,426 and $376 \mathrm{~km} \mathrm{~s}^{-1}$. For the $3-\mathrm{h} \sigma_{\mathrm{z}}^{2}$ values, the daily averages are 5.3, 1.8, 0.4 and $1.4 \mathrm{nT}^{2}$. For the $3-\mathrm{h} \sigma_{\mathrm{z}}^{2} / B_{0}^{2}$ values, they are $0.20,0.12,0.05$ and 0.09 , respectively. One can note the general decrease in the $B_{\mathrm{z}}$ variances and normalized $B_{\mathrm{Z}}$ variances with decreasing solar wind speed.

\section{Summary}

This past solar cycle minimum (SC23 minimum) has been extreme in several respects. The sunspot number $\left(R_{\mathrm{Z}}\right)$ was the lowest in recent history. Associated with this feature, Fig. 1 has shown that the duration of cycle 23 has been elongated (12.6 years) in comparison to cycles 20 through 22 (11.7, 10.3 and 9.7 years, respectively). Both the solar magnetic flux and the interplanetary field magnitude near Earth were minima about a half year after the sunspot solar minima, with the center of the minimum located somewhere during mid-2009 (Fig. 3).

The focus of this paper is the cause of low geomagnetic activity at Earth during/near the last solar minimum. We have identified and investigated in detail an interval from day 97 , 


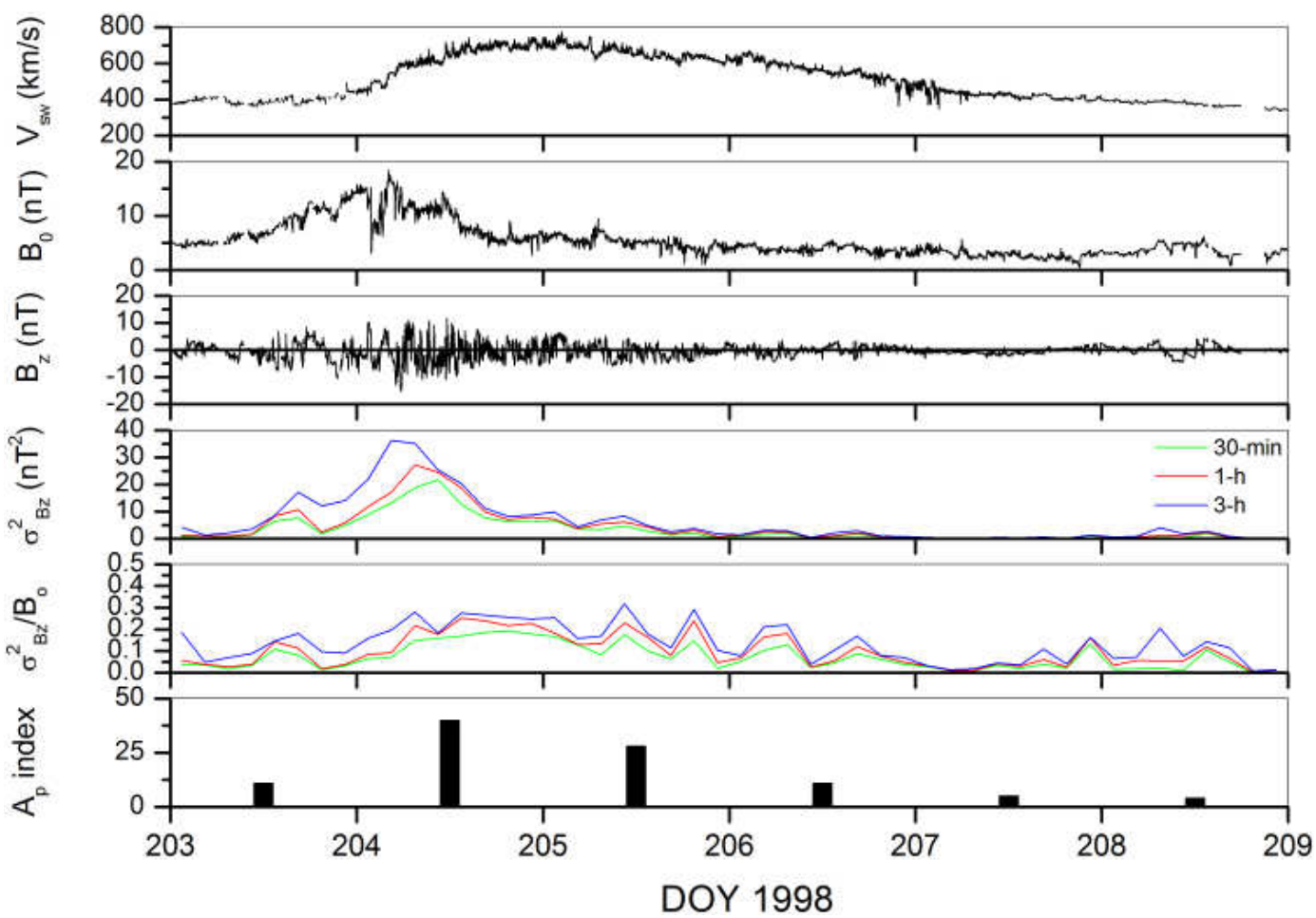

Fig. 8. A high speed stream during 1998 during the rising part of SC23. The format is the same as in Fig. 4, but the magnetic field magnitude $B_{0}$ has been added to illustrate the presence of the CIR and the effect it has on the $B_{\mathrm{Z}}$ variances and normalized $B_{\mathrm{Z}}$ variances.

2008 to day 95, 2010 (Fig. 1). This has been called MGA23 and has been shown to be characterized by decreasing solar wind peak high speed streams, decreasing IMF $B_{\mathrm{Z}}$ variances and normalized $B_{\mathrm{Z}}$ variances and ap indices (Figs. 2 and 4). During 2009, there were very few streams with peak speeds $>600 \mathrm{~km} \mathrm{~s}^{-1}$. Correspondingly the IMF $B_{\mathrm{Z}}$ variances, normalized $B_{\mathrm{Z}}$ variances and ap indices were the lowest within the interval (Tables 1 and 2).

A similar MGA interval was identified near the SC22 minimum, from day 106, 1996 to day 23, 1998. The MGA22 interval had similar solar wind, $B_{\mathrm{Z}}$ variances, normalized $B_{\mathrm{Z}}$ variances and ap index features as were present for MGA23. The solar wind peak speeds, variances and ap indices were anomalously low for a subinterval of MGA22, from 1997.2 to 1998.0 (Fig. 6).

The high level of IMF $B_{\mathrm{Z}}$ variances and normalized $B_{\mathrm{Z}}$ variances were shown to be an intrinsic part of high speed streams (Fig. 5). The southward component of the interplanetary Alfvénic fluctuations leads to magnetic reconnection at Earth and concomitant high ap values.

De Toma (2011) has indicated that during 2009 there was a disappearance of low-latitude coronal holes with only small mid-latitude coronal holes remaining. An example of a midlatitude 2009 coronal hole was shown (Fig. 7).
Peak solar wind speeds near the center of coronal holes are $\sim 750$ to $800 \mathrm{~km} \mathrm{~s}^{-1}$ with the speeds falling off gradually at the trailing edge. The $B_{\mathrm{Z}}$ variances and normalized $B_{\mathrm{Z}}$ variances are maximum near the peak in speed (neglecting the CIR portion) and decrease with decreasing speed on the trailing part of the high speed stream (Fig. 8).

\section{Conclusions}

It is well established that high speed streams and embedded Alfvénic fluctuations emanate from coronal holes and the impingement of the streams and the southward component of the Alfvénic fluctuations (IMF $B_{\mathrm{Z}}$ ) lead to magnetic reconnection and geomagnetic activity (Tsurutani et al., 1995, 2006).

The peak speed of the solar wind over a polar coronal hole has been shown to be a constant $\sim 750$ to $800 \mathrm{~km} \mathrm{~s}^{-1}$ (Phillips et al., 1994). Similar values for peak speeds of equatorial coronal holes have been shown here and in many prior publications (Tsurutani et al., 1995, 2006, 2011; Echer et al., 2011). However at the edges of high speed streams, implicitly assuming that one is not over the center of the coronal hole, the speed and the IMF variances decrease (Fig. 8).

The minima in geomagnetic activity (MGA) during the SC23 minimum and the SC22 minimum occurred well after the sunspot number minima. MGA23 occurred during 


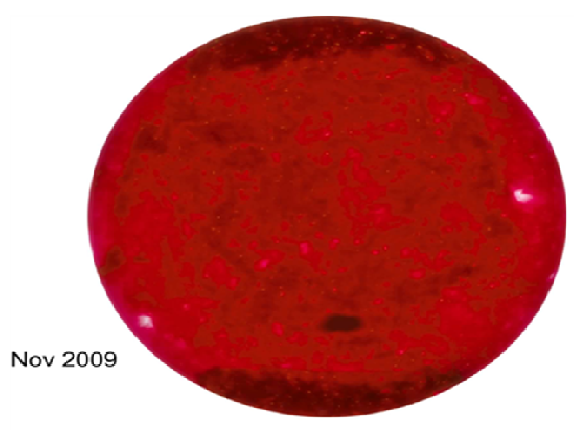

Blowup of isolated coronal hole

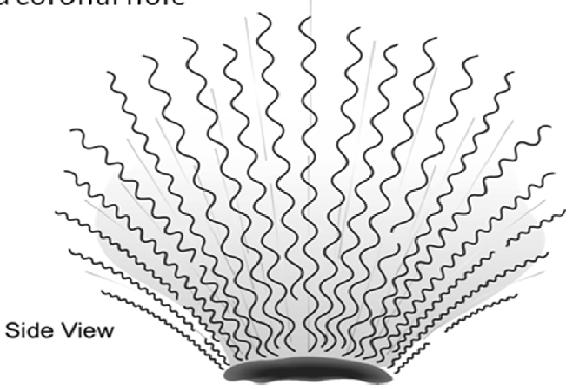

Fig. 9. The top panel shows an artist's schematic of the midlatitude coronal hole and polar coronal holes in Fig. 8. The bottom panel shows a blowup (and side view) of the midlatitude coronal hole, the solar wind velocity emanating from the hole and the Alfvén fluctuations carried by the solar wind. The solar wind speed is highest ( $\sim 750$ to $800 \mathrm{~km} \mathrm{~s}^{-1}$ ) over the center of the hole. The Alfvén wave amplitudes are the largest there. Because of superradial expansion, the solar wind speed and Alfvén wave amplitudes are diminished at the edges of the hole.

2009 and MGA22 during 1997. The MGA23 interval was characterized by the disappearance of equatorial/low latitude coronal holes, low $B_{\mathrm{Z}}$ variances and low normalized $B_{\mathrm{Z}}$ variances.

Figure 9 is a schematic that summarizes our thoughts on the solar causes of MGA23. The top panel is an artist's schematic of the midlatitude and polar coronal holes shown in Fig. 8. The bottom panel shows a blowup schematic of the solar wind speed and embedded Alfvén waves associated with a coronal hole. The solar wind speed and Alfvén wave amplitudes are maximum directly above the coronal hole. Both are diminished at the sides of the hole due to superradial expansion effects.

The midlatitude placement of the isolated coronal hole means that the maximum solar wind speed and the maximum IMF $B_{\mathrm{Z}}$ variances (and normalized $B_{\mathrm{Z}}$ variances) will not be transmitted to the Earth and its magnetosphere. Only the edges of the high speed streams and far lower $B_{\mathrm{z}}$ variances will impact the Earth, yielding far less magnetic reconnection and concomitant lower geomagnetic activity.

It should be noted that the MGA occurred during the IMF $B_{0}$ minimum interval. Therefore the causes of the MGA are multifold: the low interplanetary magnetic field magnitudes and low fluctuations of those fields (variances) are a part of the cause. Another part is the low solar wind speeds (Echer et al., 2008, has shown that it is the interplanetary electric field [ $\left.E_{\text {dawn-dusk }}=V_{\text {sw }} B_{\text {south }}\right]$ that is the geoeffective parameter). Both the low solar wind speeds and the low level of the IMF $B_{\mathrm{Z}}$ fluctuations (variances) are in turn caused by the placement of the coronal holes at midlatitudes. The picture is a complex one.

\section{Final comments}

It has been noted by Eddy (1976) that the Maunder Minimum (1645-1715) was characterized by a lack of auroral sightings. Our work here argues that the MGA23 was caused by low solar and interplanetary magnetic fields and the disappearance of equatorial/low latitude coronal holes, coincident with the presence of midlatitude coronal holes. Was the Maunder Minimum similar to the MGA23 interval but far longer in extent, or could even the midlatitude coronal holes have disappeared, leading to even lower solar wind speeds and IMF $B_{\mathrm{Z}}$ fluctuations? Could the interplanetary magnetic field magnitude have been even lower? Or perhaps both conditions existed during the Maunder Minimum? It is not certain at this time how such questions might be answered, but they are certainly of interest to space weather scientists.

Acknowledgements. Portions of this work were performed at the Jet Propulsion Laboratory, California Institute of Technology under contract with NASA. EE would like to thank the CNPq (PQ300211/2008-2) and FAPESP (2007/52533-1) agencies for financial support. WDG would like to thank the FAPESP agency (2008/06650-9) for financial support. BTT thanks INPE for logistical support during his sabbatical stay in Sao Jose dos Campos, SP, Brazil during 2 months of 2010.

Topical Editor R. Nakamura thanks E. Cliver for his help in evaluating this paper.

\section{References}

Akasofu, S.-I.: Energy coupling between the solar wind and the magnetosphere, Space Sci. Rev., 28, 121-190, 1981.

Balogh, A., Bothmer, V., Crooker, N. U., Forsyth, R. J., Gloeckler, G., Hewish, A., Hilchenbach, M., Kallenbach, R., Klecker, B., Linker, J. A., Lucek, E., Mann, G., Marsch, E., Posner, A., Richardson, I. G., Schmidt, J. M., Scholer, M., Wang, Y. M., Wimmer-Schweingruber, R. F., Aellig, M. R., Bochsler, P., Hefti, S., and Mikic, Z.: The solar origin of corotating interaction regions and their formation in the inner heliosphere - Report of Working Group 1, Space Sci. Rev., 89, 141-178, 1999.

Belcher, J. W. and Davis Jr., L.: Large-amplitude Alfvén waves in the interplanetary medium, 2, J. Geophys. Res., 76, 3534-3563, 1971.

De Toma, G.: Evolution of coronal holes and implication for high speed solar wind during the minimum between cycles 23 and 24, Solar Phys., in press, doi:10.1007/s11207-010-9677-2, 2011.

Dungey, J. W.: Interplanetary magnetic fields and the auroral zones, Phys. Rev. Lett., 6, 47-48, 1961. 
Echer, E., Gonzalez, W. D., Guarnieri, F. L., Dal Lago, A., and Vieira, L. E. A.: Introduction to space weather, Adv. Space Res., 35, 855-865, 2005.

Echer, E., Gonzalez, W. D., Tsurutani, B. T., and Gonzalez, A. L. C.: Interplanetary conditions causing intense geomagnetic storms (Dst $<-100 \mathrm{nT}$ ) during solar cycle 23 (1996 C2006), J. Geophys. Res., 113, 1-20, 2008.

Echer, E., Tsurutani, B. T., Gonzalez, W. D., and Kozyra, J. U.: High speed stream properties and related geomagnetic activity during the Whole Heliosphere Interval (WHI): 20 March to 16 April 2008, Sol. Phys., in press, doi:10.1007/s11207-011-97390, 2011.

Eddy, J. A.: The Maunder Minimum, Science, 192, 1189-1202, 1976.

Gonzalez, W. D. and Mozer, F. S.: A quantitative model for the potential resulting from reconnection with an arbitrary interplanetary magnetic field, J. Geophys. Res., 79, 4186-4194, 1974.

Gonzalez, W. D., Joselyn, J. A., Kamide, Y., Kroehl, H. W., Rostoker, G., Tsurutani, B. T., and Vasyliunas, V. M.: What is a geomagnetic storm?, J. Geophys.Res., 99, 5771-5792, 1994.

Gonzalez, W. D., Tsurutani, B. T., and Clua de Gonzalez, A. L.: Interplanetary origin of geomagnetic storms, Space Sci. Rev., 88, 529-562, 1999.

Hathaway, D. H.: The Solar Cycle, Living Rev. Solar Phys., 7, 165, 2010.

Perrault, P. D. and Akasofu, S.-I.: A study of geomagnetic storms, Geophys. J. R. Astron. Soc., 54, 547-573, 1978.

Phillips, J. L., Balogh, A., Bame, S. J., Goldstein, B. E., Gosling, J. T., Hoeksema, J. T., McComas, D. J., Neugebauer, M., Sheeley Jr., N. R., and Wang, Y. M.: Ulysses at $50^{\circ}$ south: Constant immersion in the high speed solar wind, Geophys. Res. Lett., 21, 1105-1108, 1994

Rostoker, G.: Geomagnetic Indices, Rev. Geophys., 10, 935-950, 1972.

Scherrer, P. H., Wilcox, J. M., Svalgaard, L., Duvall, T. L., Dittmer, P. H., and Gustafson, E. K.: The mean magnetic field of the sun: Observations at Stanford, Solar Phys., 54, 353-361, 1977.
Solomon, S. C., Woods, T. N., Didkovsky, L. V., Emmert, J. T., and Qian, L.: Anomalously low solar extreme-ultraviolet irradiance and thermospheric density during solar minimum, Geophys. Res. Lett., 37, L16103, doi:10.1029/2010GL044468, 2010.

Tsurutani, B. T. and Gonzalez, W. D.: The cause of high-intensity long-duration continuous AE activity (HILDCAAs): Interplanetary Alfvén wave trains, Planet. Space Sci., 35, 405-412, 1987.

Tsurutani, B. T. and Meng, C.-I.: Interplanetary magnetic field variations and substorms, J. Geophys. Res., 77, 2964-2970, 1972.

Tsurutani, B. T., Smith, E. J., Pyle, K. R., and Simpson, J. A.: Energetic protons accelerated at corotating shocks: Pioneer 10 and 11 observations from 1 to 6 AU, J. Geophys. Res., 87, 7389-7404, 1982.

Tsurutani, B. T., Gonzalez, W. D., Tang, F., Akasofu, S.-I., and Smith, E. J.: Origin of interplanetary southward magnetic fields responsible for major magnetic storms near solar maximum (1978-1979), J. Geophys. Res., 93, 8519-8531, 1988.

Tsurutani, B. T., Gonzalez, W. D., Gonzalez, A. L. C., Tang, F., Arballo, J. K., and Okada, M.: Interplanetary origin of geomagnetic activity in the declining phase of the solar cycle, J. Geophys. Res., 100, 21717-21733, 1995.

Tsurutani, B. T. and Gonzalez, W. D.: The interplanetary causes of magnetic storms: A review, in: Magnetic Storms, edited by: Tsurutani, B. T., Gonzalez, W. D., Kamide, Y., and Arballo, J. K., Amer. Geophys. Un. Press, Wash. D.C., 98, 77-89, 1997.

Tsurutani, B. T., Gonzalez, W. D., Gonzalez, A. L. C., Guarnieri, F. L., Gopalswamy, N., Grande, M., Kamide, Y., Kasahara, Y., Lu, G., Mann, I., McPherron, R. L., Soraas, F., and Vasyliunas, V. M.: Corotating solar wind streams and recurrent geomagnetic activity: A review, J. Geophys. Res., 111, A07S01, doi:10.1029/2005JA011273, 2006.

Tsurutani, B. T., Echer, E., Guarnieri, F. L., and Gonzalez, W. D.: The properties of two solar wind high speed streams and related geomagnetic activity during the declining phase of solar cycle 23, J. Atmos. Solar-Terr. Phys., 73, 164, doi:10.1016/j.jastp.2010.04.003, 2011. 